\title{
3D Structure of the Long Fimbriae of the Gram-Positive Bacteria Streptococcus parasanguinis
}

\author{
Chul Ho Park, Carsten Peters, Michael Radermacher, Teresa Ruiz \\ Department of Molecular Physiology and Biophysics, University of Vermont, Burlington, VT
}

Adhesion is important from both the physiological and the pathophysiological aspect of bacterial colonization. Streptococcus parasanguinis, a Gram-positive bacterium, is one of the primary colonizers of the oral cavity and provides the foundation for a complex multi species biofilm, known as dental plaque [1]. In addition, as opportunistic pathogens, these bacteria can adhere to native and prosthetic heart valves leading to the development of endocarditis [2]. S. parasanguinis adhesion to host surfaces is mediated by long proteinaceous appendages of $\sim 0.6 \mu \mathrm{m}$ in length, which have been denominated long fimbriae [3]. Since their initial discovery on the surface of S. parasanguinis, these fimbriae have been observed in other members of the streptococcal and staphylococcal families (e.g. S. gordonii, S. pneumoniae, S. aureus, S. epidermidis). Fap1 (fimbriae-associated protein 1), a serine-rich glycoprotein protein of $\sim 200 \mathrm{kDa}$ molecular mass, is the main structural component of the long fimbriae. Even though a wealth of information is available at the genetic and biochemical level, structural studies of Fap1 related fimbriae are in their infancy due to the large subunit size ( $>$ $200 \mathrm{kDa}$ ) and the difficulty in isolating large quantities of the individual fimbrial subunits required for X-ray or NMR studies.

We have used 3D electron microscopy from negatively stained preparations of whole mount wild type bacteria to obtain structural information of the fimbriae on the bacterial surface. Good results have been obtained with preparations in 1\% uranyl acetate (UA), 2\% phosphotunstic acid, $\mathrm{pH} 7$ (PTA) and 2\% methyl amine tungstate $\mathrm{pH} 7.5$ (MAT). In all the stained preparations, the fimbriae have a diameter of $\sim 5.5 \mathrm{~nm}$ and show a strong repeat at $6.5 \mathrm{~nm}$ both in Real and Fourier space. Average power spectra from many fimbrial segments reveal additional repeats at $32 \mathrm{~nm}, 10.5 \mathrm{~nm}$, and $4 \mathrm{~nm}$ under all staining conditions. Combining the largest observed repeat with the subunit molecular weight and the fimbrial diameter we proposed a model of the subunit arrangement in the fimbriae. Our data correlates well with the fimbriae being composed of two strands of Fap1 subunits winding around each other.

We have carried out studies in the three different stains to determine the structure of the Fap1 fimbriae, since in spite of the strong structural similarities we also observed specific characteristics that were stain dependent. Namely, at neutral pH (PTA and MAT) the fimbriae were close together forming rafts, and at low $\mathrm{pH}$ (UA) were separated along their length while the apical ends of several fimbriae came together to form aggregates. To calculate the $3 \mathrm{D}$ reconstructions, fimbrial regions have been extracted from the CTF corrected images following strict criteria: the fimbriae should be straight; they should not overlap other fimbriae and should be separated by at least two fimbrial diameters from its nearest neighbor. The boxed regions were subdivided into as many images as possible of $\sim 55 \mathrm{~nm}$ in length. An averaged image was calculated and used to create an initial reference volume using back-projection algorithms. Subsequently, all the images were translationally/rotationally aligned to the reference volume using Radon transform algorithms [5], resulting in random tilt angles during the first round of alignment. The aligned images were used to create a new reference volume. This alignment procedure was repeated several times, adapting the 
alignment parameters in each round. The repeats observed in the 2D images as well as additional fine structure can be observed in the 3D volume. Currently, we are trying to interpret and understand how the single Fap1 subunit is incorporated into the fimbriae structure.

\section{References}

[1] E. H. Froeliger, P.M. Fives-Taylor (2001). Infect Immun. 69, 2512-19.

[2] H.B. Viscount, C.L. Munro, D. Burnette-Curley, D.L. Peterson, and F.L. Macrina (1997). Infect Immun. 65(3), 994-1002.

[3] H. Wu, K.P. Mintz, M.. Ladha, P.M. Fives-Taylor (1998). Mol Microbiol. 28, 487-500.

[4] L. Oligion, P.M. Fives-Taylor (1993). Infect Immun. 61, 1016-22.

[5] M. Radermacher (1997). Scanning Microscopy. 11, 171-77.

[6] This work was supported by NIH grant R01 DE017474 (awarded to T.R) and benefited from developments supported by NIH grant R01GM078202 (awarded to MR)
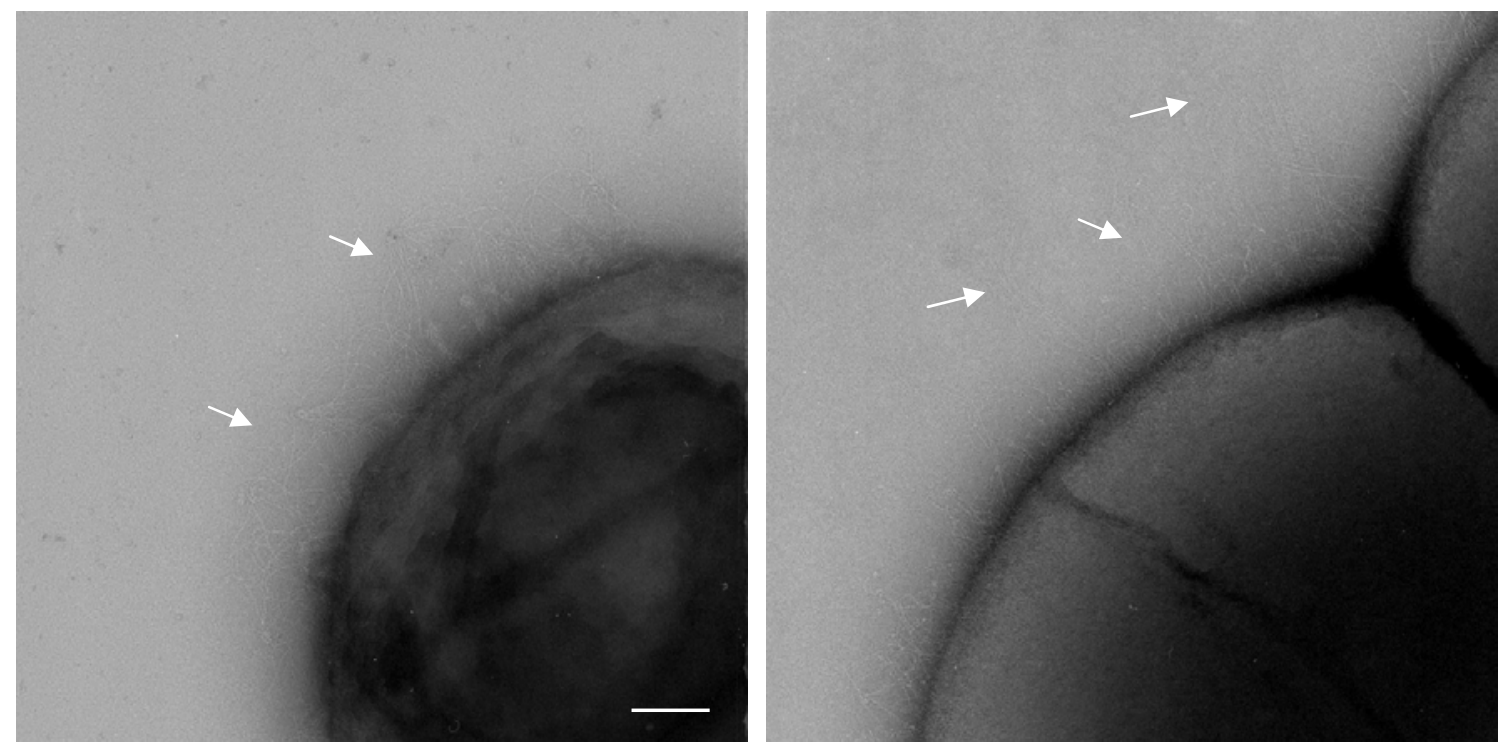

Figure 1. Electron micrographs of whole-mount S. parasanguinis stained by UA (left) and PTA (right). Arrows point to fimbriae. Scale bar $=100 \mathrm{~nm}$. 